NOTICE: this is the author's version of a work that was accepted for publication in Automatica. Changes resulting from the publishing process, such as peer review, editing, corrections, structural formatting, and other quality control mechanisms may not be reflected in this document. Changes may have been made to this work since it was submitted for publication. A definitive version was subsequently published in Automatica., Vol. 48, no.7 (2012). DOI: 10.1016/j.automatica.2011.11.013 


\title{
Formation Control of Underactuated Ships with Elliptical Shape Approximation and Limited Communication Ranges
}

\author{
K. D. Do \\ School of Mechanical Engineering, The University of Western Australia \\ 35 Stirling Highway, Crawley, WA 6009, Australia
}

\begin{abstract}
Based on the recent theoretical development for formation control of multiple fully actuated agents with an elliptical shape in Do (2011), this paper develops distributed controllers that force a group of $N$ underactuated ships with limited communication ranges to perform a desired formation, and guarantee no collisions between any ships in the group. The ships are first fitted to elliptical disks for solving collision avoidance. A coordinate transformation is then proposed to introduce an additional control input, which overcomes difficulties caused by underactuation and off-diagonal terms in the system matrices. The control design relies on potential functions with the separation condition between elliptical disks and the smooth or $p$-times differentiable step functions embedded in.
\end{abstract}

Keywords: Underactuated ships, formation control, elliptical disks, collision avoidance, potential functions

\section{Introduction}

Formation control of a group of underactuated ships is a hard and challenging problem due to difficulties in controlling each single ship while requiring to perform cooperative tasks for the group. The reader is referred to Do and Pan (2009) and references therein for various control methods for single underactuated ships. There are several approaches mentioned below to formation control design for underactuated ships.

The leader-follower approach plus the Lyapunov and sliding mode methods were used in Lapierre et al. (2003), Fahimi (2007), Schoerling et al. (2010), Cui et al. (2010) to design cooperative controllers for a group of underactuated vessels. A combination of line-of-sight path-following and nonlinear synchronization strategies was studied in Borhaug et al. (2006), Borhaug et al. (2010) to make a group of underactuated vessels asymptotically follow a given straight-line path with a given forward speed profile. In Dong and Farrell (2008), see also Dong and Farrell (2009), nontrivial coordinate changes, graph theory, and stability theory of linear time-varying systems were used to design cooperative control laws for underactuated vessels to perform a geometric pattern.

In the above papers, collision avoidance between vessels was not considered even though a collision between vessels can cause a catastrophic failure. Embedding a collision avoidance algorithm in a formation control design for underactuated ships is difficult due to the stability problem of zero dynamics of the un-actuated degree of freedom. Moreover, ships usually have a long and narrow shape. Fitting them to circular disks results in a problem of the large conservative area defined as the difference between the areas enclosed by the circle and the ellipse. Using the result in Section 1 in Do (2011), it can be shown that the conservative area is proportional to square of the difference between the length and the width of the ship. In addition, an elliptical fitting covers a circular one by setting the semi-axes of the bounding ellipse equal, but not vice versa.

Email address: duc@mech.uwa.edu.au (K. D. Do) a)

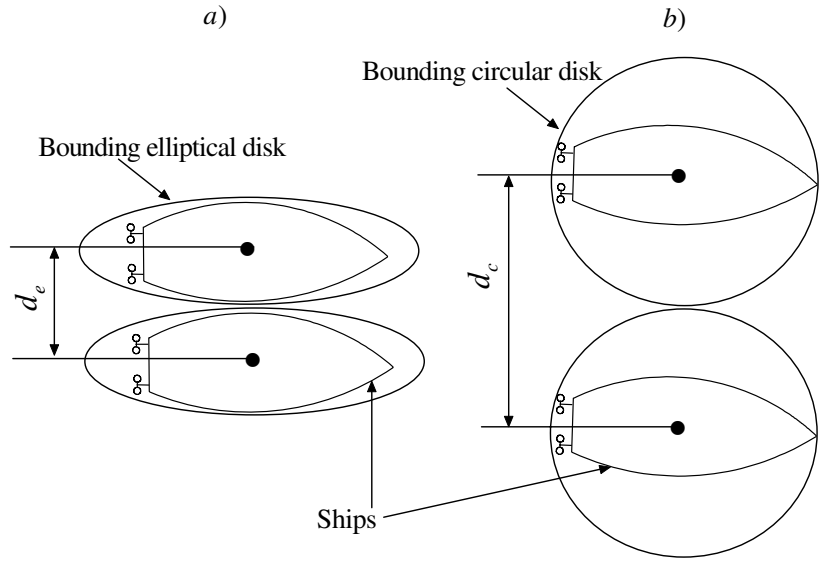

Figure 1: Comparison of sway distance when bounding ships by a) elliptical disks and b) circular disks

In practice, there are cases where it is necessary to navigate a group of underactuated ships moving in a formation that requires the distance in the sway direction between the ships in the group as short as possible. An example is a refueling scenario between two ships. As illustrated in Figure 1, when bounding each ship with a long and narrow shape by an elliptical disk the distance $d_{e}$ (in the sway direction between two ships) is much shorter than the distance $d_{c}$ when bounding each ship by a circular disk.

In comparison with formation control of fully actuated agents with an elliptical shape in Do (2011), formation control design for elliptical ships is difficult due to the underactuation problem. It is not straightforward to combine the techniques developed for stabilization and trajectory tracking control of underactuated ships in Do and Pan (2009) and references therein with the formation control design method in Do (2011) to design a formation control system for underactuated ships. This is due to the fact that the techniques in Do and Pan (2009) and references therein use the heading angle as an 
immediate control to control the sway displacement. Consequently, it is not an easy task to embed the collision avoidance in a proper potential function for formation control design, see also Do (2008) for a discussion where formation control of mobile robots was addressed.

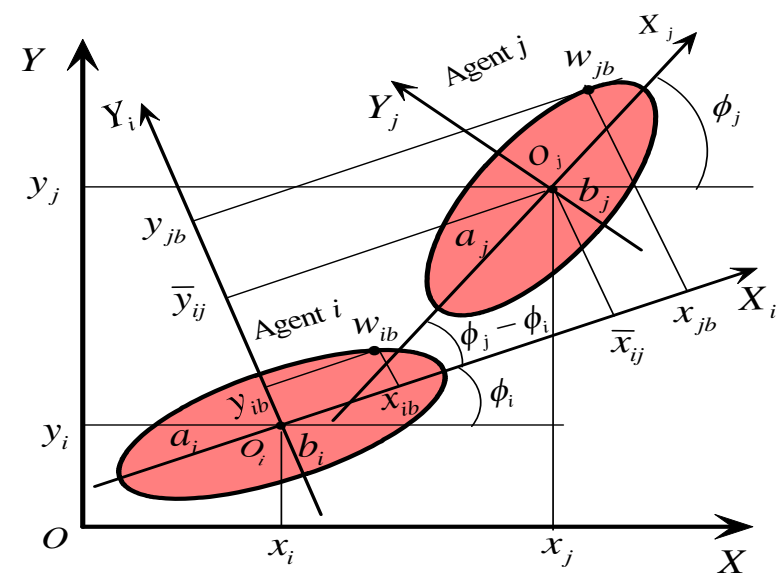

Figure 2: Two elliptical disks and their coordinates

The aforementioned observations motivate contributions of this paper on design of formation control algorithms for underactuated ships with an elliptical shape and limited communication ranges. The paper's contributions include: 1) a way to embed the condition for separation between two elliptical disks proposed in Do (2011) in a new potential function for deriving formation control algorithms, see Section 4.1 ; 2) a design of formation controller, see Section 4; and 3) stability analysis of critical points of the closed loop system, see Appendix Appendix A.

\section{Preliminaries}

\subsection{Separation condition between two elliptical disks}

This section presents a condition for separation of two elliptical disks applicable for embedding collision avoidance in the formation control design later.

Lemma 2.1. Consider two elliptical disks $i$ and $j$, of which bounding ellipses have semi-axes of $\left(a_{i}, b_{i}\right)$ and $\left(a_{j}, b_{j}\right)$, are centered at $\left(x_{i}, y_{i}\right)$ and $\left(x_{j}, y_{j}\right)$, and have heading angles of $\phi_{i}$ and $\phi_{j}$, respectively, see Figure 2. Define the generalized distance $\Delta_{i j}$ between the elliptical disks $i$ and $j$ as

$$
\Delta_{i j}=\sqrt{\frac{1}{2}\left(\left\|\boldsymbol{Q}_{i j} \overline{\boldsymbol{p}}_{i j}\right\|^{2}+\left\|\boldsymbol{Q}_{j i} \overline{\boldsymbol{p}}_{j i}\right\|^{2}\right)},
$$

where $\overline{\boldsymbol{p}}_{i j}=\left[\begin{array}{ll}\bar{x}_{i j} & \bar{y}_{i j}\end{array}\right]^{T}$,

$$
\boldsymbol{Q}_{i j}=\left[\begin{array}{rr}
-\frac{\kappa_{i j} \cos \left(\alpha_{i j}\right)}{a_{i}\left(\kappa_{i j}+\hat{a}_{j}^{2}\right)} & -\frac{\kappa_{i j} \sin \left(\alpha_{i j}\right)}{b_{i}\left(\kappa_{i j}+\hat{a}_{j}^{2}\right)} \\
\frac{\kappa_{i j} \sin \left(\alpha_{i j}\right)}{a_{i}\left(\kappa_{i j}+\hat{b}_{j}^{2}\right)} & -\frac{\kappa_{i j} \cos \left(\alpha_{i j}\right)}{b_{i}\left(\kappa_{i j}+\hat{b}_{j}^{2}\right)}
\end{array}\right],
$$

and $\kappa_{i j}$ is the unique solution of the following equation:

$$
F\left(\kappa_{i j}\right):=\left(\frac{\hat{a}_{j} \hat{x}_{i j}}{\kappa_{i j}+\hat{a}_{j}^{2}}\right)^{2}+\left(\frac{\hat{b}_{j} \hat{y}_{i j}}{\kappa_{i j}+\hat{b}_{j}^{2}}\right)^{2}-1=0 .
$$

All the variables $\hat{a}_{j}, \hat{b}_{j}, \bar{x}_{i j}, \bar{y}_{i j}, \hat{x}_{i j}, \hat{y}_{i j}$, and $\alpha_{i j}$ are given by:

$$
\begin{aligned}
& \hat{a}_{j}=1 / \sqrt{T_{a}}, \hat{b}_{j}=1 / \sqrt{T_{b}}, \\
& {\left[\begin{array}{c}
\hat{x}_{i j} \\
\hat{y}_{i j}
\end{array}\right]=\left[\begin{array}{cc}
-\frac{1}{a_{i}} \cos \left(\alpha_{i j}\right) & -\frac{1}{b_{i}} \sin \left(\alpha_{i j}\right) \\
\frac{1}{a_{i}} \sin \left(\alpha_{i j}\right) & -\frac{1}{b_{i}} \cos \left(\alpha_{i j}\right)
\end{array}\right] \overline{\boldsymbol{p}}_{i j},} \\
& \alpha_{i j}=2 \arctan \left(\frac{2\left(T_{11} T_{12}+T_{21} T_{22}\right)}{T_{11}^{2}+T_{21}^{2}+T_{12}^{2}+T_{22}^{2}}\right),
\end{aligned}
$$

with

$$
\begin{aligned}
& T_{a}=\left(T_{11}^{2}+T_{21}^{2}\right) \cos ^{2}\left(\alpha_{i j}\right)+\left(T_{11} T_{12}+T_{21} T_{22}\right) \times \\
& \sin \left(2 \alpha_{i j}\right)+\left(T_{12}^{2}+T_{22}^{2}\right) \sin ^{2}\left(\alpha_{i j}\right), \\
& T_{b}=\left(T_{11}^{2}+T_{21}^{2}\right) \sin ^{2}\left(\alpha_{i j}\right)-\left(T_{11} T_{12}+T_{21} T_{22}\right) \times \\
& \sin \left(2 \alpha_{i j}\right)+\left(T_{12}^{2}+T_{22}^{2}\right) \cos ^{2}\left(\alpha_{i j}\right), \\
& T_{11}= \frac{a_{i}}{a_{j}} \cos \left(\phi_{i j}\right), \quad T_{12}=-\frac{b_{i}}{a_{j}} \sin \left(\phi_{i j}\right), \\
& T_{21}= \frac{a_{i}}{b_{j}} \sin \left(\phi_{i j}\right), \quad T_{22}=\frac{b_{i}}{b_{j}} \cos \left(\phi_{i j}\right), \\
& x_{i j}= x_{i}-x_{j}, \quad y_{i j}=y_{i}-y_{j}, \phi_{i j}=\phi_{i}-\phi_{j}, \\
& {\left[\bar{x}_{i j}, \bar{y}_{i j}\right]^{T}=\boldsymbol{R}_{i}\left[x_{i j}, y_{i j}\right]^{T}, }
\end{aligned}
$$

where $\boldsymbol{R}_{i}=-\boldsymbol{R}^{-1}\left(\phi_{i}\right)$ with $\boldsymbol{R}(\bullet)$ the rotational matrix of $\bullet$. The matrix $\boldsymbol{Q}_{j i}$ and the vector $\overline{\boldsymbol{p}}_{j i}$ are defined accordingly. The two elliptical disks are separated if

$$
\Delta_{i j}>1
$$

Proof. See Do (2011).

\section{2. p-times differentiable step function}

This section defines and constructs $p$-times differentiable or smooth step functions. These functions are to be embedded into a potential function to avoid discontinuities in the control law due to the ships' communication limited ranges.

Definition 2.1. A scalar function $h(x, a, b)$ is said to be a $p$ times differentiable step function if it has the properties:
1) $h(x, a, b)=0, \forall x \in(-\infty, a]$,
2) $h(x, a, b)=1, \forall x \in[b, \infty)$
3) $0<h(x, a, b)<1, \forall x \in(a, b)$,
4) $h(x, a, b)$ is p times differentiable,

where $p$ is a positive integer, $x \in \mathbb{R}$, and $a$ and $b$ are constants such that $a<b$. Moreover, if the function $h(x, a, b)$ is infinite times differentiable with respect to $x$, then it is said to be a smooth step function.

Lemma 2.2. Let the scalar function $h(x, a, b)$ be defined as

$$
h(x, a, b)=\frac{\int_{a}^{x} f(\tau-a) f(b-\tau) d \tau}{\int_{a}^{b} f(\tau-a) f(b-\tau) d \tau},
$$

with $a$ and $b$ constants such that $a<b$, and the function $f(y)$ defined as

$$
f(y)=0 \text { if } y \leq 0, f(y)=g(y) \text { if } y>0,
$$

where the function $g(y)$ has the following properties

a) $g(\tau-a) g(b-\tau)>0, \quad \forall \tau \in(a, b)$,

b) $g(y)$ is $p$ times differentiable,

c) $\lim _{y \rightarrow 0^{+}} \frac{\partial^{k} g(y)}{\partial y^{k}}=0, k=1, \ldots, p-1$, 
with $p$ a positive integer. Then $h(x, a, b)$ is a p-times differentiable step function. Moreover, if $g(y)$ in (9) is replaced by $g(y)=e^{-1 / y}$ then $h(x, a, b)$ is a smooth step function.

\section{Proof. See Do (2007).}

3. Problem statement 3.1. Ship dynamics

We consider a group of $N$ underactuated ships. The ship $i$ equipped with a pair of propellers or water jets, for all $i \in \mathbb{N}$ with $\mathbb{N}$ the set of all ships, has the dynamics, Fossen (2002):

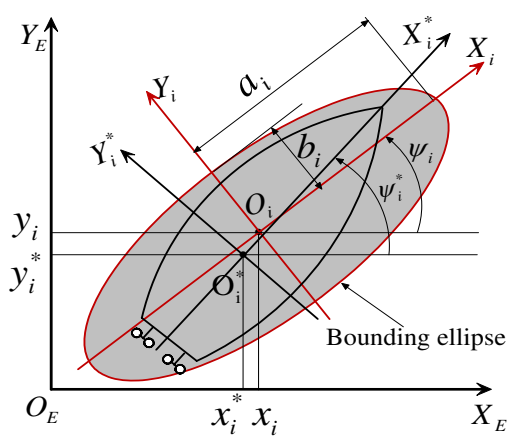

Figure 3: Ship's coordinates

$$
\begin{aligned}
& \dot{\boldsymbol{\eta}}_{i}^{*}=\boldsymbol{J}\left(\psi_{i}^{*}\right) \boldsymbol{v}_{i}, \\
& \boldsymbol{M}_{i} \dot{\boldsymbol{v}}_{i}=-\boldsymbol{C}_{i}\left(\boldsymbol{v}_{i}\right) \boldsymbol{v}_{i}-\boldsymbol{D}_{i}\left(\boldsymbol{v}_{i}\right) \boldsymbol{v}_{i}+\boldsymbol{\tau}_{i}+\boldsymbol{J}^{T}\left(\psi_{i}^{*}\right) \boldsymbol{d}_{i},
\end{aligned}
$$

where $\boldsymbol{\eta}_{i}^{*}=\left[x_{i}^{*} y_{i}^{*} \psi_{i}^{*}\right]^{T}$ denotes the ship position $\left(x_{i}^{*}, y_{i}^{*}\right)$ and yaw angle $\psi_{i}^{*}$ coordinated in the earth-fixed frame; $\boldsymbol{v}_{i}=$ $\left[\begin{array}{lll}u_{i} & v_{i} & r_{i}\end{array}\right]^{T}$ denotes the ship velocities coordinated in the bodyfixed frame; $\boldsymbol{d}_{i}=\left[\begin{array}{lll}d_{1 i} & d_{2 i} & d_{3 i}\end{array}\right]^{T}$ denotes external constant forces due to wind and ocean currents coordinated in the earthfixed frame; $\tau_{i}=\left[\begin{array}{lll}\tau_{u i} & 0 & \tau_{r i}\end{array}\right]^{T}$ denotes the surge force $\tau_{u i}$, and the yaw moment $\tau_{r i}$; and

$$
\begin{aligned}
& \boldsymbol{J}\left(\psi_{i}^{*}\right)=\left[\begin{array}{cc}
\boldsymbol{R}\left(\psi_{i}^{*}\right) & \mathbf{0}_{2 \times 1} \\
\mathbf{0}_{1 \times 2} & 1
\end{array}\right], \boldsymbol{D}_{i}\left(\boldsymbol{v}_{i}\right)=-\left[\begin{array}{ccc}
d_{11 i} & 0 & 0 \\
0 & d_{22 i} & d_{23 i} \\
0 & d_{32 i} & d_{33 i}
\end{array}\right], \\
& \boldsymbol{M}_{i}=\left[\begin{array}{ccc}
m_{11 i} & 0 & 0 \\
0 & m_{22 i} & m_{23 i} \\
0 & m_{23 i} & m_{33 i}
\end{array}\right], \boldsymbol{C}_{i}\left(v_{i}\right)=\left[\begin{array}{ccc}
0 & 0 & c_{13 i} \\
0 & 0 & c_{23 i} \\
-c_{13 i} & -c_{23 i} & 0
\end{array}\right],
\end{aligned}
$$

with

$$
\begin{aligned}
& m_{11 i}=m_{i}-X_{\dot{u} i}, m_{22 i}=m_{i}-Y_{\dot{v} i}, m_{23 i}=m_{i} x_{g i}-Y_{\dot{r} i}, \\
& m_{33 i}=I_{z i}-N_{\dot{r} i}, c_{13 i}=-m_{22 i} v_{i}-m_{23 i} r_{i}, c_{23 i}=m_{11 i} u_{i}, \\
& d_{11 i}=-\left(X_{u i}+X_{u|u| i}\left|u_{i}\right|\right), d_{22 i}=-\left(Y_{v i}+Y_{|v| v i}\left|v_{i}\right|+Y_{|r| v i}\left|r_{i}\right|\right), \\
& d_{23 i}=-\left(Y_{r i}+Y_{|v| r i}\left|v_{i}\right|+Y_{|r| r i}\left|r_{i}\right|\right), d_{32 i}=-\left(N_{v i}+N_{|v| v i}\left|v_{i}\right|+\right. \\
& \left.N_{|r| v i}\left|r_{i}\right|\right), d_{33 i}=-\left(N_{r i}+N_{|v| r i}\left|v_{i}\right|+N_{|r| r i}\left|r_{i}\right|\right) .
\end{aligned}
$$

In (13), $m_{i}$ is the mass of the ship; $I_{z i}$ is the ship's inertia about the $Z_{i}^{*}$-axis of the body-fixed frame; $x_{g i}^{*}$ is the $X_{i}^{*}$-coordinate of the ship center of gravity in the body-fixed frame; the other symbols are hydrodynamic derivatives, SNAME (1950).

\subsection{Transformation of ship dynamics}

We combine the coordinate change on page 168 in Do and Pan (2009) to get around the difficulty caused by the term $m_{23 i}$ and the transverse function approach in Morin and Samson (2003) to create an additional control input for solving the underactuated problem, see also Do (2010). We introduce the coordinate change:

$$
\begin{aligned}
& {\left[\begin{array}{l}
x_{i} \\
y_{i}
\end{array}\right]=\left[\begin{array}{c}
x_{i}^{*}+\varepsilon_{i} \cos \left(\psi_{i}^{*}\right) \\
y_{i}^{*}+\varepsilon_{i} \sin \left(\psi_{i}^{*}\right)
\end{array}\right]+\boldsymbol{R}\left(\psi_{i}\right)\left[\begin{array}{l}
f_{1 i}\left(\alpha_{i}\right) \\
f_{2 i}\left(\alpha_{i}\right)
\end{array}\right],} \\
& \psi_{i}=\psi_{i}^{*}-f_{3 i}\left(\alpha_{i}\right), \\
& \bar{v}_{i}=v_{i}+\varepsilon_{i} r_{i},
\end{aligned}
$$

where $\varepsilon_{i}=m_{23 i} / m_{22 i}, f_{l i}\left(\alpha_{i}\right), l=1,2,3$, are to be determined later. With (14), the ship's dynamics (11) is rewritten as:

$$
\begin{aligned}
& {\left[\begin{array}{c}
\dot{x}_{i} \\
\dot{y}_{i}
\end{array}\right]=\boldsymbol{A}_{i}\left[\begin{array}{c}
u_{i} \\
\dot{\alpha}_{i}
\end{array}\right]+\left[\begin{array}{c}
-\bar{v}_{i} \sin \left(\psi_{i}^{*}\right) \\
\bar{v}_{i} \cos \left(\psi_{i}^{*}\right)
\end{array}\right]+\boldsymbol{R}^{\prime}\left(\psi_{i}\right)\left[\begin{array}{c}
f_{1 i}\left(\alpha_{i}\right) \\
f_{2 i}\left(\alpha_{i}\right)
\end{array}\right] \times} \\
& \quad\left(r_{i}-f_{3 i}^{\prime}\left(\alpha_{i}\right) \dot{\alpha}_{i}\right), \\
& \dot{\psi}_{i}=r_{i}-f_{3 i}^{\prime}\left(\alpha_{i}\right) \dot{\alpha}_{i}, \\
& \dot{u}_{i}=\varphi_{u i}+\frac{1}{m_{11 i}} \tau_{u i}+\frac{1}{m_{11 i}}\left(d_{1 i} \cos \left(\psi_{i}^{*}\right)+d_{2 i} \sin \left(\psi_{i}^{*}\right)\right), \\
& \dot{\bar{v}}_{i}=\varphi_{v i}+\frac{m_{23 i}}{m_{22 i}} \varphi_{r i}+\frac{1}{m_{22 i}}\left(-d_{1 i} \sin \left(\psi_{i}^{*}\right)+d_{2 i} \cos \left(\psi_{i}^{*}\right)\right), \\
& \dot{r}_{i}=\varphi_{r i}+\frac{m_{22 i}}{\Delta_{i}} \tau_{r i}+\frac{m_{23 i}}{\Delta_{i}}\left(d_{1 i} \sin \left(\psi_{i}^{*}\right)-d_{2 i} \cos \left(\psi_{i}^{*}\right)\right)+\frac{m_{22 i}}{\Delta_{i}} d_{3 i},
\end{aligned}
$$

where $\dot{\alpha}_{i}$ is referred to as an additional control, and

$$
\begin{aligned}
\boldsymbol{A}_{i}= & {\left.\left[\begin{array}{c}
\cos \left(\psi_{i}^{*}\right) \\
\sin \left(\psi_{i}^{*}\right)
\end{array}\right] \boldsymbol{R}\left(\psi_{i}\right)\left[\begin{array}{c}
f_{1 i}^{\prime}\left(\alpha_{i}\right) \\
f_{2 i}^{\prime}\left(\alpha_{i}\right)
\end{array}\right]\right], \Delta_{i}=m_{22 i} m_{33 i}-m_{23 i}^{2}, } \\
\varphi_{u i}= & \frac{m_{22 i}}{m_{11 i}} v_{i} r_{i}+\frac{m_{23 i}}{m_{11 i}} r_{i}^{2}-\frac{d_{11 i}}{m_{11 i}} u_{i}, \\
\varphi_{v i}= & -\frac{m_{11 i}}{m_{22 i}} u_{i} r_{i}-\frac{d_{22 i}}{m_{22 i}} v_{i}-\frac{d_{23 i}}{m_{22 i}} r_{i}, \\
\varphi_{r i}= & \frac{m_{11 i} m_{22 i}-m_{22 i}^{2}}{\Delta_{i}} u_{i} v_{i}+\frac{m_{11 i} m_{23 i}-m_{23 i} m_{22 i}}{\Delta_{i}} u_{i} r_{i}- \\
& \frac{m_{22 i}}{\Delta_{i}}\left(d_{33 i} r_{i}+d_{32 i} v_{i}\right)+\frac{m_{23 i}}{\Delta_{i}}\left(d_{23 i} r_{i}+d_{22 i} v_{i}\right),
\end{aligned}
$$

and $f_{l i}^{\prime}\left(\alpha_{i}\right)=\frac{\partial f_{l i}\left(\alpha_{i}\right)}{\partial\left(\alpha_{i}\right)}, l=1,2,3$ and $\boldsymbol{R}^{\prime}\left(\psi_{i}\right)=\frac{\partial \boldsymbol{R}\left(\psi_{i}\right)}{\partial \psi_{i}}$. We now choose $f_{l i}$ such that $f_{l i}$ are bounded, differentiable and make the matrix $\boldsymbol{A}_{i}$ invertible for all $\psi_{i}^{*} \in \mathbb{R}$ and $\alpha_{i} \in \mathbb{R}$ as

$$
\begin{aligned}
& f_{1 i}=\epsilon_{1 i} \sin \left(\alpha_{i}\right) \frac{\sin \left(f_{3 i}\right)}{f_{3 i}}, f_{2 i}=\epsilon_{2 i} \sin \left(\alpha_{i}\right) \frac{1-\cos \left(f_{3 i}\right)}{f_{3 i}}, \\
& f_{3 i}=\epsilon_{3 i} \cos \left(\alpha_{i}\right),
\end{aligned}
$$

where the constants $\epsilon_{1 i}$ and $\epsilon_{2 i}$ are chosen such that $\epsilon_{1 i}>0$, $\epsilon_{2 i}=\epsilon_{1 i}$, and $0<\epsilon_{3 i}<\frac{\pi}{2}$. With the choice of $f_{l i}$ in (17), a calculation shows that $\left|f_{1 i}\right| \leq \epsilon_{1 i}, \quad\left|f_{2 i}\right| \leq \epsilon_{1 i}, \quad\left|f_{3 i}\right| \leq \epsilon_{3 i}$ and $\operatorname{det}\left(\boldsymbol{A}_{i}\right) \leq-\frac{\epsilon_{1 i}}{\epsilon_{3 i}}\left(1-\cos \left(\epsilon_{3 i}\right)\right), \forall \alpha_{i} \in \mathbb{R}$. Due to the $\bar{v}_{i}$-dynamics in (15), the constants $\epsilon_{l i}$ need to satisfy an additional condition to prevent instability of the $\bar{v}_{i}$-dynamics. This will be detailed after the formation control is designed.

\subsection{Bounding the ship by an elliptical disk}

From (14) and (17), a calculation shows that

$$
\begin{array}{r}
\left\|\left(x_{i}-x_{i}^{*}, y_{i}-y_{i}^{*}\right)\right\| \leq \\
\varepsilon_{i}+\sqrt{\epsilon_{1 i}^{2}+\epsilon_{2 i}^{2}}, \\
\left|\psi_{i}-\psi_{i}^{*}\right| \leq \epsilon_{3 i} .
\end{array}
$$

Therefore, we can bound the ship by an elliptical disk with a heading angle of $\psi_{i}$ and the center

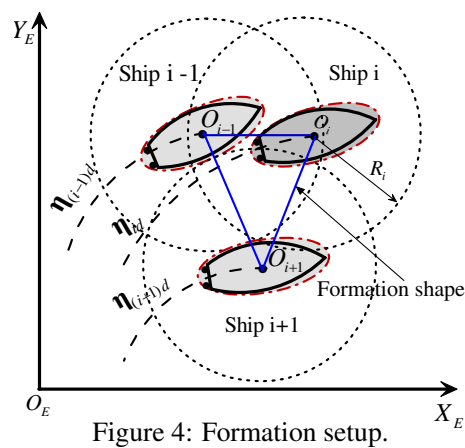
at the point $O_{i}$ coordinated at $\left(x_{i}, y_{i}\right)$, see Figure 3 . The semiaxes, $a_{i}$ and $b_{i}$, of the bounding elliptical disk can be calculated from the ship's length, width, the point $O_{i}^{*}$ coordinated at $\left(x_{i}^{*}, y_{i}^{*}\right)$ and the heading angle $\psi_{i}^{*}$ using the bounds (18). 


\subsection{Formation control objective}

To design a formation controller, there is a need of specifying a common goal for the ships, some communication between them, and their initial position and orientation. Hence, the following assumption is imposed on the reference trajectories, communication, and initial conditions between the ships:

\section{Assumption 3.1.}

1) The reference trajectory, $\boldsymbol{\eta}_{i d}\left(s_{i d}\right)=\left[x_{i d}\left(s_{i d}\right) y_{i d}\left(s_{i d}\right)\right.$ $\left.\psi_{i d}\left(s_{i d}\right)\right]^{T}, i=1, \ldots, N$ with $s_{i d}$ the parameter of $\boldsymbol{\eta}_{i d}\left(s_{i d}\right)$, for each ship $i, i \in \mathbb{N}$, has bounded derivatives and satisfies

$$
\Delta_{i j d}>1
$$

where $\Delta_{i j d}$ is given in (1) with $\boldsymbol{\eta}_{i}$ and $\boldsymbol{\eta}_{j}$ replaced by $\boldsymbol{\eta}_{i d}$ and $\boldsymbol{\eta}_{j d}$, respectively, and $s_{i d}=s_{j d}$.

2) The ships $i$ and $j$ have circular communication areas centered at the points $O_{i}$ and $O_{j}$, and with radii of $R_{i}$ and $R_{j}$, see Figure 4 . The radii $R_{i}$ and $R_{j}$ satisfy the condition

$$
\Delta_{i j R}^{m}>1,
$$

where $\Delta_{i j R}$ is the greatest lower bound of $\Delta_{i j}$ when the ships $i$ and $j$ are within their communication ranges, i.e.,

$$
\begin{gathered}
\Delta_{i j R}^{m}=\inf \left(\Delta_{i j}\right) \quad \text { s.t. } \quad \psi_{i j} \in \mathbb{R}, \bar{x}_{i j}^{2}+\bar{y}_{i j}^{2}=\min \left(R_{i}^{2}, R_{j}^{2}\right), \\
\forall(i, j) \in \mathbb{N}, j \neq i .
\end{gathered}
$$

3) The ship i broadcasts $\boldsymbol{\eta}_{i}$ and $\boldsymbol{\eta}_{i d}$ in its communication area. Moreover, the ship i can receive $\boldsymbol{\eta}_{j}$ and $\boldsymbol{\eta}_{j d}$ broadcasted by other ships $j, j \in \mathbb{N}, j \neq i$ if the points $O_{j}$ of these ships are in the communication area of the ship $i$.

4) At the initial time $t_{0} \geq 0$, all the ships are sufficiently far away from each other, i.e., they satisfy the condition:

$$
\Delta_{i j}\left(t_{0}\right)>1
$$

where $\Delta_{i j}\left(t_{0}\right)$ is given in (1) with $\boldsymbol{\eta}_{i}=\boldsymbol{\eta}_{i}\left(t_{0}\right)$ and $\boldsymbol{\eta}_{j}=\boldsymbol{\eta}_{j}\left(t_{0}\right)$.

Formation Control Objective 3.1. Under Assumption 3.1, design the control inputs $\tau_{u i}$ and $\tau_{\text {ri }}$ for each ship $i$ such that the trajectory $\boldsymbol{\eta}_{i}$ tracks the reference trajectory $\boldsymbol{\eta}_{i d}$ while avoids collisions with all other ships. In addition, $s_{i d}$ and $\dot{s}_{i d}$ of $\boldsymbol{\eta}_{i d}$ are to approach the common reference trajectory parameter $s_{\text {od }}$ and its rate $\dot{s}_{\text {od }}$, i.e.,

$$
\begin{aligned}
& \lim _{t \rightarrow \infty}\left(\left(\boldsymbol{\eta}_{i}(t)-\boldsymbol{\eta}_{i d}(t)\right),\left(s_{i d}(t)-s_{o d}(t)\right),\left(\dot{s}_{i d}(t)-\dot{s}_{o d}(t)\right)\right)=0, \\
& \Delta_{i j}(t)>1, \forall(i, j) \in \mathbb{N}, i \neq j, t \geq t_{0} \geq 0 .
\end{aligned}
$$

\section{Formation Control Design}

The system (15) is of a strict feedback form. Therefore, we will apply Lyapunov's method and the backstepping technique Krstic et al. (1995) to design the controls $\tau_{u i}$ and $\tau_{r i}$.

\subsection{Stage I}

Define the following errors

$$
\begin{aligned}
& u_{i e}=u_{i}-\varpi_{u i}, \\
& r_{i e}=r_{i}-\varpi_{r i},
\end{aligned}
$$

where $\varpi_{u i}$ and $\varpi_{r i}$ are the virtual controls of $u_{i}$ and $r_{i}$, respectively. This stage designs $\varpi_{u i}, \varpi_{r i}$, and $\dot{\alpha}_{i}$ to achieve the task of trajectory tracking and collision avoidance. As motivated by the work in Do (2011), we consider the following potential function:

$$
\varphi_{I}=\sum_{i=1}^{N}\left(\gamma_{i}+\beta_{i}\right) .
$$

The goal function $\gamma_{i}$ for the ship $i$ puts penalty on the tracking errors between $\boldsymbol{\eta}_{i}$ and $\boldsymbol{\eta}_{i d}$, and is chosen as:

$$
\gamma_{i}=0.5\left(\boldsymbol{p}_{i}-\boldsymbol{p}_{i d}\right)^{T} \boldsymbol{K}_{1}\left(\boldsymbol{p}_{i}-\boldsymbol{p}_{i d}\right)+0.5 k_{2}\left(\psi_{i}-\psi_{i d}\right)^{2},
$$

where $\boldsymbol{p}_{i}=\left[\begin{array}{ll}x_{i} & y_{i}\end{array}\right]^{T}, \boldsymbol{p}_{i d}=\left[\begin{array}{ll}x_{i d} & y_{i d}\end{array}\right]^{T}$, and $\boldsymbol{K}_{1}$ is a $2 \times 2$ diagonal positive definite matrix and $k_{2}$ is a positive constant.

The collision avoidance function $\beta_{i}$ prevents collisions between the ship $i$ and other ships, and is chosen as:

$$
\beta_{i}=\frac{1}{2} \sum_{j \in \mathbb{N}_{i}} \beta_{i j},
$$

where $\mathbb{N}_{i}$ is the set that contains all the ships in the group except for the ship $i$. The pairwise collision avoidance function $\beta_{i j}$ between the ships $i$ and $j$ is a function of $\chi_{i j}$, with $\chi_{i j}$ given by

$$
\chi_{i j}=0.5\left(\sqrt{\Delta_{i j}^{2}+\epsilon^{2}}-\sqrt{1+\epsilon^{2}}\right)^{2},
$$

where $\epsilon$ is a positive constant, and has the properties:

1) $\beta_{i j}=0, \beta_{i j}^{\prime}=0, \beta_{i j}^{\prime \prime}=0, \forall \chi_{i j} \in\left[\min \left(\chi_{i j R}^{m}, \chi_{i j d}\right), \infty\right)$,

2) $\beta_{i j}>0, \beta_{i j}^{\prime}<0, \forall \chi_{i j} \in\left(0, \min \left(\chi_{i j R}^{m}, \chi_{i j d}\right)\right)$,

3) $\lim _{\chi_{i j} \rightarrow 0} \beta_{i j}=\infty, \lim _{\chi_{i j} \rightarrow 0} \beta_{i j}^{\prime}=-\infty$,

4) $\beta_{i j}$ is at least three times differentiable,

5) $\beta_{i j} \leq \mu_{1 i j},\left|\beta_{i j}^{\prime}\right| \leq \mu_{2 i j},\left|\beta_{i j}^{\prime \prime}\right| \leq \mu_{3 i j}, \quad \forall 0<\chi_{i j} \leq \mu_{4 i j}$,

for all $(i, j) \in \mathbb{N}$ and $i \neq j$, where $\beta_{i j}^{\prime}=\frac{\partial \beta_{i j}}{\partial \chi_{i j}} ; \beta_{i j}^{\prime \prime}=\frac{\partial^{2} \beta_{i j}}{\partial \chi_{i j}^{2}}$; $\mu_{l i j}, l=1, \ldots, 4$ are positive constants; and $\chi_{i j d}$ and $\chi_{i j R}^{m}$ are $\chi_{i j}$ given in (28) with $\Delta_{i j}$ replaced by $\Delta_{i j d}$ and $\Delta_{i j R}^{m}$, respectively.

Remark 4.1. Properties 1) - 3) imply that $\beta_{i}$ is positive definite, is equal to zero when $\boldsymbol{\eta}_{i}-\boldsymbol{\eta}_{i d}=0$, and is equal to infinity when a collision between the ship $i$ and any other ships occurs. Also, Property 1) ensures that the collision avoidance between the ships $i$ and $j$ is only taken into account when they are in their communication areas. Properties 3) and 5) are used to prove stability of the closed loop system. Property 4) allows us to use control design and stability analysis for continuous systems found in Khalil (2002) to handle the collision avoidance problem under the ships' limited communication ranges.

Based on the $p$-times differentiable step function in Section 2.2 , we can find many functions that satisfy all properties listed in (29). As an example, we will use the following function $\beta_{i j}$ in the rest of the paper:

$$
\beta_{i j}=k_{i j}\left(1-h_{i j}\left(\chi_{i j}, a_{i j}, b_{i j}\right)\right) / \chi_{i j}
$$

where $k_{i j}$ is a positive constant, $\chi_{i j}$ is given by (28) and $h_{i j}\left(\chi_{i j}, a_{i j}, b_{i j}\right)$ is a $p$-times differentiable step function with $p \geq 3$ and the function $g(y)$ taken as $g(y)=y^{p}$. The constants $a_{i j}$ and $b_{i j}$ are chosen such that

$$
0<a_{i j}<b_{i j} \leq \chi_{i j d}-\mu_{i j d},
$$


where $\mu_{i j d}$ is a positive constant.

To design the virtual controls $\varpi_{u i}$ and $\varpi_{r i}$ and the additional control $\dot{\alpha}_{i}$, we differentiate both sides of (25) along the solutions of (24) and the first three equations of (15) to obtain

$$
\begin{gathered}
\dot{\varphi}_{I}=\sum_{i=1}^{N}\left[( \boldsymbol { K } _ { 1 } ( \boldsymbol { p } _ { i } - \boldsymbol { p } _ { i d } ) + \sum _ { j \neq i } \beta _ { j i } ^ { \prime } \boldsymbol { \Gamma } _ { i j } ) ^ { T } \left(\boldsymbol{A}_{i}\left[\begin{array}{c}
u_{i e}+\varpi_{u i} \\
\dot{\alpha}_{i}
\end{array}\right]+\right.\right. \\
\left.\boldsymbol{R}^{\prime}\left(\psi_{i}\right)\left[\begin{array}{c}
f_{1 i} \\
f_{2 i}
\end{array}\right]\left(r_{i e}+\varpi_{r i}-f_{3 i}^{\prime} \dot{\alpha}_{i}\right)+\left[\begin{array}{c}
-\bar{v}_{i} \sin \left(\psi_{i}^{*}\right) \\
\bar{v}_{i} \cos \left(\psi_{i}^{*}\right)
\end{array}\right]-\dot{\boldsymbol{p}}_{i d}\right)+ \\
\left.\left(k_{2}\left(\psi_{i}-\psi_{i d}\right)+\sum_{j \neq i} \beta_{j i}^{\prime} \Xi_{i j}\right)\left(r_{i e}+\varpi_{r i}-f_{3 i}^{\prime} \dot{\alpha}_{i}-\dot{\psi}_{i d}\right)+\Omega_{i d} \dot{s}_{i d}\right],
\end{gathered}
$$

where we dropped the argument $\alpha_{i}$ of $f_{l i}$ and $f_{l i}^{\prime}$, and

$$
\begin{aligned}
F_{i j} & =\left(\frac{\hat{a}_{j} \hat{x}_{i j}}{\kappa_{i j}+\hat{a}_{j}^{2}}\right)^{2}+\left(\frac{\hat{b}_{j} \hat{y}_{i j}}{\kappa_{i j}+\hat{b}_{j}^{2}}\right)^{2}, \\
\boldsymbol{G}_{i j} & =\left(\boldsymbol{Q}_{i j} \overline{\boldsymbol{p}}_{i j}\right)^{T}\left(\boldsymbol{Q}_{i j}-\left(\frac{\partial F_{i j}}{\partial \kappa_{i j}}\right)^{-1} \frac{\partial \boldsymbol{Q}_{i j}}{\partial \kappa_{i j}} \overline{\boldsymbol{p}}_{i j}\left(\frac{\partial F_{i j}}{\partial \overline{\boldsymbol{p}}_{i j}}\right)^{T}\right), \\
H_{i j} & =\left(\boldsymbol{Q}_{i j} \overline{\boldsymbol{p}}_{i j}\right)^{T}\left(\frac{\partial \boldsymbol{Q}_{i j}}{\partial \psi_{i j}} \overline{\boldsymbol{p}}_{i j}-\left(\frac{\partial F_{i j}}{\partial \kappa_{i j}}\right)^{-1} \frac{\partial \boldsymbol{Q}_{i j}}{\partial \kappa_{i j}} \overline{\boldsymbol{p}}_{i j} \frac{\partial F_{i j}}{\partial \psi_{i j}}\right) . \\
\boldsymbol{\Gamma}_{i j} & =\left(-\boldsymbol{G}_{i j} \boldsymbol{R}_{i}^{T}+\boldsymbol{G}_{j i} \boldsymbol{R}_{j}^{T}\right), \Xi_{i j}=\left(\boldsymbol{G}_{i j} \boldsymbol{S} \boldsymbol{p}_{i j}+H_{i j}-H_{j i}\right), \\
\Omega_{i d} & =\sum_{j \neq i} \beta_{j i}^{\prime} \boldsymbol{\Gamma}_{i j}^{T}\left[x_{i d}^{\prime} y_{i d}^{\prime}\right]^{T}+\sum_{j \neq i} \beta_{j i}^{\prime} \Xi_{i j} \psi_{i d}^{\prime},
\end{aligned}
$$

with $S=\left[\begin{array}{cc}0 & 1 \\ -1 & 0\end{array}\right], \boldsymbol{p}_{i j}=\boldsymbol{p}_{i}-\boldsymbol{p}_{j}, x_{i d}^{\prime}=\frac{\partial x_{i d}}{\partial s_{i d}}, y_{i d}^{\prime}=\frac{\partial y_{i d}}{\partial s_{i d}}$, and $\psi_{i d}^{\prime}=\frac{\partial \psi_{i d}}{\partial s_{i d}}$. Note that $\frac{\partial F_{i j}}{\partial \kappa_{i j}}$ is always nonzero, see Proof of Lemma 2.1 in Do (2011).

From (32), we design the virtual controls $\varpi_{u i}$ and $\varpi_{r i}$, and the additional control $\dot{\alpha}_{i}$ as follows

$$
\begin{aligned}
& {\left[\begin{array}{c}
\varpi_{u i} \\
\dot{\alpha}_{i}
\end{array}\right]=\boldsymbol{A}_{i}^{-1}\left(-c_{1} \boldsymbol{\Phi}\left(\boldsymbol{\Omega}_{p i}\right)-\left[-\bar{v}_{i} \sin \left(\psi_{i}^{*}\right) \bar{v}_{i} \times\right.\right.} \\
& \left.\left.\cos \left(\psi_{i}^{*}\right)\right]^{T}-\boldsymbol{R}^{\prime}\left(\psi_{i}\right)\left[f_{1 i} f_{2 i}\right]^{T}\left(-c_{1} \Phi\left(\Omega_{\psi_{i}}\right)+\dot{\psi}_{i d}\right)+\dot{\boldsymbol{p}}_{i d}\right), \\
& \varpi_{r i}=-c_{1} \Phi\left(\Omega_{\psi_{i}}\right)+f_{3 i}^{\prime} \dot{\alpha}_{i}+\dot{\psi}_{i d},
\end{aligned}
$$

where $c_{1}$ is a positive constant, and

$$
\boldsymbol{\Omega}_{p i}=\boldsymbol{K}_{1}\left(\boldsymbol{p}_{i}-\boldsymbol{p}_{i d}\right)+\sum_{j \neq i} \beta_{j i}^{\prime} \boldsymbol{\Gamma}_{i j}, \Omega_{\psi_{i}}=k_{2}\left(\psi_{i}-\psi_{i d}\right)+\sum_{j \neq i} \beta_{j i}^{\prime} \Xi_{i j} .
$$

The vector function $\boldsymbol{\Phi}\left(\boldsymbol{\Omega}_{p i}\right)=\left[\Phi\left(\Omega_{1 p i}\right) \Phi\left(\Omega_{2 p i}\right)\right]^{T}$ with $\boldsymbol{\Omega}_{p i}=$ $\left[\Omega_{1 p i} \Omega_{2 p i}\right]^{T}$. The function $\Phi(x)$ satisfies

$$
\begin{aligned}
& \text { 1) }|\Phi(x)| \leq M_{1}, \Phi(0)=0, x \Phi(x)>0 \text { if } x \neq 0, \\
& \text { 2) } \Phi(-x)=-\Phi(x),(x-y)[\Phi(x)-\Phi(y)] \geq 0, \\
& \text { 3) }\left|\frac{\Phi(x)}{x}\right| \leq M_{2},\left|\frac{\partial \Phi(x)}{\partial x}\right| \leq M_{3},\left.\frac{\partial \Phi(x)}{\partial x}\right|_{x=0}=1,
\end{aligned}
$$

for all $x \in \mathbb{R}, y \in \mathbb{R}$, where $M_{1}, M_{2}, M_{3}$ are positive constants. The update law, $\dot{\boldsymbol{s}}_{i d}$, is designed as:

$$
\dot{s}_{i d}=h\left(\chi_{i j}, a_{i j d}, b_{i j d}\right)\left(-k_{i d}\left(s_{i d}-s_{o d}\right)+\dot{s}_{o d}\right),
$$

where $k_{i d}$ is a positive constant, and $s_{i d}\left(t_{0}\right)=s_{o d}\left(t_{0}\right)$; $h\left(\chi_{i j}, b_{i j}, \chi_{i j R}^{M}\right)$ is a $p$-times differentiable step function with $p \geq 3$. The constants $a_{i j d}$ and $b_{i j d}$ are chosen as

$$
a_{i j d}=b_{i j}, b_{i j d}<\min \left(\chi_{i j R}^{M}, \chi_{i j d}\right),
$$

where $b_{i j}$ is given in (31), and $\chi_{i j R}^{M}$ is $\chi_{i j}$ given in (28) with $\Delta_{i j}$ replaced by its least upper bound value, $\Delta_{i j R}^{M}$, when the ships $i$ and $j$ are in their communication ranges, i.e.,

$$
\Delta_{i j R}^{M}=\sup \left(\Delta_{i j}\right) \text { s.t. } \psi_{i j} \in \mathbb{R}, \bar{x}_{i j}^{2}+\bar{y}_{i j}^{2}=\min \left(R_{i}^{2}, R_{j}^{2}\right) .
$$

The choice (38) results in $\beta_{i j}^{\prime} h\left(\chi_{i j}, b_{i j}, \chi_{i j R}^{M}\right)=0 \Rightarrow \Omega_{i d} \dot{s}_{i d}=$ 0 , and ensures that $h\left(\Delta_{i j}, a_{i j d}, b_{i j d}\right)$ tends to 1 when $\chi_{i j}$ tends to $b_{i j d}$, which is smaller than $\chi_{i j d}$. This means that $s_{i d}$ and $\dot{s}_{i d}$ are to approach $s_{o d}$ and $\dot{s}_{o d}$ as required.

Remark 4.2. In (35), $\boldsymbol{\Omega}_{p i}$ and $\Omega_{\psi_{i}}$ consist of the gradient of $\gamma_{i}$ responsible for trajectory tracking plus the gradient of $\beta_{i}$ responsible for collision avoidance tasks. Moreover, $\varpi_{u i}, \varpi_{r i}$, and $\dot{\alpha}_{i}$ are differentiable and depend on only $\boldsymbol{\eta}_{i}, \boldsymbol{\eta}_{i d}$, and $\boldsymbol{\eta}_{j}$, $\boldsymbol{\eta}_{j d}$ of other ships $j$ if these ships $j$ are communicating with the ship i due to Property 1) of $\beta_{i j}$ in (29).

Substituting (34) and (37) into (32) gives

$$
\begin{aligned}
\dot{\varphi}_{I}= & \sum_{i=1}^{N}\left[-c_{1} \boldsymbol{\Omega}_{p i}^{T} \mathbf{\Phi}\left(\boldsymbol{\Omega}_{p i}\right)-c_{1} \Omega_{\psi_{i}} \Phi\left(\Omega_{\psi_{i}}\right)+\right. \\
& \left.\mathbf{\Omega}_{p i}^{T} \boldsymbol{A}_{i}\left[u_{i e} 0\right]^{T}+\left(\boldsymbol{\Omega}_{p i}^{T} \boldsymbol{R}^{\prime}\left(\psi_{i}\right)\left[f_{1 i} f_{2 i}\right]^{T}+\Omega_{\psi_{i}}\right) r_{i e}\right] .
\end{aligned}
$$

\subsection{Stage II}

In this stage, we design the actual controls $\tau_{u i}$ and $\tau_{r i}$. Since $\boldsymbol{d}_{i}$ is unknown, we apply Lemma 3.1 in Do (2010) to (11) to estimate the disturbance vector $\boldsymbol{d}_{i}$ as follows:

$$
\begin{aligned}
\hat{\boldsymbol{d}}_{i}= & \boldsymbol{\xi}_{i}+\boldsymbol{K}_{0 i} \boldsymbol{J}^{-T}\left(\psi_{i}^{*}\right) \boldsymbol{M}_{i} \boldsymbol{v}_{i}, \\
\dot{\boldsymbol{\xi}}_{i}= & -\boldsymbol{K}_{0 i} \boldsymbol{\xi}_{i}-\boldsymbol{K}_{0 i}\left(\dot{\boldsymbol{J}}^{-T}\left(\psi_{i}^{*}\right) \boldsymbol{M}_{i} \boldsymbol{v}_{i}+\boldsymbol{J}^{-T}\left(\psi_{i}^{*}\right) \boldsymbol{M}_{i}\left(\boldsymbol{M}_{i}^{-1} \times\right.\right. \\
& \left.\left.\left(-\boldsymbol{C}_{i}\left(\boldsymbol{v}_{i}\right) \boldsymbol{v}_{i}-\boldsymbol{D}_{i}\left(\boldsymbol{v}_{i}\right) \boldsymbol{v}_{i}+\boldsymbol{\tau}_{i}\right)\right)+\boldsymbol{K}_{0 i} \boldsymbol{J}^{-T}\left(\psi_{i}^{*}\right) \boldsymbol{M}_{i} \boldsymbol{v}_{i}\right),
\end{aligned}
$$

where $\boldsymbol{K}_{0 i}$ is a positive definite matrix. The above disturbance observer results in the observer error dynamics $\dot{\boldsymbol{d}}_{i e}=-\boldsymbol{K}_{0 i} \boldsymbol{d}_{i e}$, where $\boldsymbol{d}_{i e}=\left[\begin{array}{lll}d_{1 i e} & d_{2 i e} & d_{3 i e}\end{array}\right]^{T}=\boldsymbol{d}_{i}-\hat{\boldsymbol{d}}_{i}$. To design $\tau_{u i}$ and $\tau_{r i}$, we consider the following Lyapunov function candidate

$$
\varphi_{I I}=\varphi_{I}+\frac{1}{2} \sum_{i=1}^{N}\left(u_{i e}^{2}+r_{i e}^{2}\right)
$$

By differentiating both sides of (42) along the solutions of (24), (15) and (34), the controls $\tau_{u i}$ and $\tau_{r i}$ are chosen as:

$$
\begin{gathered}
\tau_{u i}=m_{11 i}\left(-c_{2} u_{i e}-\mathbf{\Omega}_{p i}^{T} \boldsymbol{A}_{i}[10]^{T}-\left[\varphi_{u i}+\frac{1}{m_{11 i}}\left(\hat{d}_{1 i} \cos \left(\psi_{i}^{*}\right)+\right.\right.\right. \\
\left.\hat{d}_{2 i} \sin \left(\psi_{i}^{*}\right)\right)-\left(\frac{\partial \varpi_{u i}}{\partial \boldsymbol{p}_{i}}\right)^{T} \dot{\boldsymbol{p}}_{i}-\left(\frac{\partial \varpi_{u i}}{\partial \boldsymbol{p}_{i d}}\right)^{T} \dot{\boldsymbol{p}}_{i d}-\left(\frac{\partial \varpi_{u i}}{\partial \dot{\boldsymbol{p}}_{i d}}\right)^{T} \ddot{\boldsymbol{p}}_{i d}- \\
\frac{\partial \varpi_{u i}}{\partial \alpha_{i}} \dot{\alpha}_{i}-\frac{\partial \varpi_{u i}}{\partial \psi_{i}} \dot{\psi}_{i}-\frac{\partial \varpi_{u i}}{\partial \psi_{i d}} \dot{\psi}_{i d}-\frac{\partial \varpi_{u i}}{\partial \dot{\psi}_{i d}} \ddot{\psi}_{i d}-\frac{\partial \varpi_{u i}}{\partial \bar{v}_{i}}\left(\varphi_{v i}+\right. \\
\left.\frac{m_{23 i}}{m_{22 i}} \varphi_{r i}+\frac{-\hat{d}_{1 i} \sin \left(\psi_{i}^{*}\right)+\hat{d}_{2 i} \cos \left(\psi_{i}^{*}\right)}{m_{22 i}}\right)-\sum_{j \in \mathbb{N}_{i}}\left(\left(\frac{\partial \varpi_{u i}}{\partial \boldsymbol{p}_{j}}\right)^{T} \dot{\boldsymbol{p}}_{j}+\right. \\
\left.\left.\left.\frac{\partial \varpi_{u i}}{\partial \alpha_{j}} \dot{\alpha}_{j}+\frac{\partial \varpi_{u i}}{\partial \psi_{j}} \dot{\psi}_{j}\right)\right]\right),
\end{gathered}
$$




$$
\begin{gathered}
\tau_{r i}=\frac{\Delta_{i}}{m_{22 i}}\left(-c_{2} r_{i e}-\left(\boldsymbol{\Omega}_{p i}^{T} \boldsymbol{R}^{\prime}\left(\psi_{i}\right)\left[f_{1 i} f_{2 i}\right]^{T}+\Omega_{\psi_{i}}\right)-\left[\varphi_{r i}+\right.\right. \\
\frac{m_{23 i}}{\Delta_{i}}\left(\hat{d}_{1 i} \sin \left(\psi_{i}^{*}\right)-\hat{d}_{2 i} \cos \left(\psi_{i}^{*}\right)\right)+\frac{m_{22 i}}{\Delta_{i}} \hat{d}_{3 i}-\left(\frac{\partial \varpi_{r i}}{\partial \boldsymbol{p}_{i}}\right)^{T} \dot{\boldsymbol{p}}_{i}- \\
\left(\frac{\partial \varpi_{r i}}{\partial \boldsymbol{p}_{i d}}\right)^{T} \dot{\boldsymbol{p}}_{i d}-\left(\frac{\partial \varpi_{r i}}{\partial \dot{\boldsymbol{p}}_{i d}}\right)^{T} \ddot{\boldsymbol{p}}_{i d}-\frac{\partial \varpi_{r i}}{\partial \alpha_{i}} \dot{\alpha}_{i}-\frac{\partial \varpi_{r i}}{\partial \psi_{i}} \dot{\psi}_{i}-\frac{\partial \varpi_{r i}}{\partial \psi_{i d}} \dot{\psi}_{i d} \\
-\frac{\partial \varpi_{r i}}{\partial \dot{\psi}_{i d}} \ddot{\psi}_{i d}-\frac{\partial \varpi_{r i}}{\partial \bar{v}_{i}}\left(\varphi_{v i}+\frac{m_{23 i}}{m_{22 i}} \varphi_{r i}+\frac{-\hat{d}_{1 i} \sin \left(\psi_{i}^{*}\right)}{m_{22 i}}+\right. \\
\left.\left.\left.\frac{\hat{d}_{2 i} \cos \left(\psi_{i}^{*}\right)}{m_{22 i}}\right)-\sum_{j \in \mathbb{N}_{i}}\left(\left(\frac{\partial \varpi_{r i}}{\partial \boldsymbol{p}_{j}}\right)^{T} \dot{\boldsymbol{p}}_{j}+\frac{\partial \varpi_{r i}}{\partial \alpha_{j}} \dot{\alpha}_{j}+\frac{\partial \varpi_{r i}}{\partial \psi_{j}} \dot{\psi}_{j}\right)\right]\right),
\end{gathered}
$$

where $c_{2}$ is a positive constant. With the choice of $\tau_{u i}$ and $\tau_{r i}$ given in (43), the derivative of $\varphi_{I I}$ is

$$
\begin{aligned}
\dot{\varphi}_{I I}= & \sum_{i=1}^{N}\left[-c_{1} \boldsymbol{\Omega}_{p i}^{T} \boldsymbol{\Phi}\left(\boldsymbol{\Omega}_{p i}\right)-c_{1} \Omega_{\psi_{i}} \Phi\left(\Omega_{\psi_{i}}\right)-c_{2} u_{i e}^{2}-c_{2} r_{i e}^{2}+\right. \\
& \left.u_{i e}\left(\frac{\vartheta_{1 i e}}{m_{11 i}}-\frac{\partial \varpi_{u i}}{\partial \bar{v}_{i}} \frac{\vartheta_{2 i e}}{m_{22 i}}\right)-r_{i e}\left(\frac{m_{23 i}}{\Delta_{i}} \vartheta_{2 i e}+\frac{\partial \varpi_{r i}}{\partial \bar{v}_{i}} \frac{\vartheta_{3 i e}}{m_{22 i}}\right)\right],
\end{aligned}
$$

where $\vartheta_{1 i e}=d_{1 i e} \cos \left(\psi_{i}^{*}\right)+d_{2 i e} \sin \left(\psi_{i}^{*}\right), \vartheta_{2 i e}=-d_{1 i e} \sin \left(\psi_{i}^{*}\right)+$ $d_{2 i e} \cos \left(\psi_{i}^{*}\right), \vartheta_{3 i e}=d_{1 i e} \sin \left(\psi_{i}^{*}\right)-d_{3 i e} \cos \left(\psi_{i}^{*}\right)$, and we have used $\dot{\varphi}_{I}$ in (40). The formation control design has been completed and results in the closed loop system:

$$
\begin{aligned}
& \dot{\boldsymbol{p}}_{i}=-c_{1} \boldsymbol{\Phi}\left(\boldsymbol{\Omega}_{p i}\right)+\boldsymbol{A}_{i}\left[u_{i e} 0\right]^{T}+\boldsymbol{R}^{\prime}\left(\psi_{i}\right)\left[f_{1 i} f_{2 i}\right]^{T} r_{i e}+\dot{\boldsymbol{p}}_{i d}, \\
& \dot{\psi}_{i}=-c_{1} \Phi\left(\Omega_{\psi_{i}}\right)+r_{i e}+\dot{\psi}_{i d}, \\
& \dot{u}_{i e}=-c_{2} u_{i e}-\boldsymbol{\Omega}_{p i}^{T} \boldsymbol{A}_{i}\left[\begin{array}{ll}
1 & 0
\end{array}\right]^{T}+\frac{\vartheta_{1 i e}}{m_{11 i}}-\frac{\partial \varpi_{u i}}{\partial \bar{v}_{i}} \frac{\vartheta_{2 i e}}{m_{22 i}}, \\
& \dot{r}_{i e}=-c_{2} r_{i e}-\frac{m_{23 i} \vartheta_{2 i e}}{\Delta_{i}}+\frac{\partial \varpi_{r i}}{\partial \bar{v}_{i}} \frac{\vartheta_{3 i e}}{m_{22 i}}-\boldsymbol{\Omega}_{p i}^{T} \boldsymbol{R}^{\prime}\left(\psi_{i}\right)\left[f_{1 i} f_{2 i}\right]^{T}-\Omega_{\psi_{i}}, \\
& \dot{\boldsymbol{d}}_{i e}=-\boldsymbol{K}_{0 i} \boldsymbol{d}_{i e}, \\
& \dot{\bar{v}}_{i}=\varphi_{v i}+\frac{m_{23 i}}{m_{22 i}} \varphi_{r i}+\frac{1}{m_{22 i}}\left(-d_{1 i} \sin \left(\psi_{i}^{*}\right)+d_{2 i} \cos \left(\psi_{i}^{*}\right)\right),
\end{aligned}
$$

for all $i \in \mathbb{N}$. We now present the main result of our paper in the following theorem.

Theorem 4.1. Under Assumption 3.1, the controls $\tau_{u i}$ and $\tau_{r i}$ given in (43) together with the disturbance observer given in (41) for the ship $i$ solve the formation control objective as long as the design constants $\epsilon_{l i}, l=1,2,3$ are chosen such that

$$
\begin{aligned}
& \frac{Y_{|v| v i}}{m_{22 i}}+\frac{m_{11 i}}{m_{22 i}} \frac{\epsilon_{1 i}+\epsilon_{2 i}}{\operatorname{det}\left(\boldsymbol{A}_{i}\right)}+\frac{\epsilon_{3 i}\left(\lambda_{1 i}+4 \lambda_{2 i}\right)}{\operatorname{det}\left(\boldsymbol{A}_{i}\right)} \leq-\lambda_{0 i}, \\
& \epsilon_{1 i}>0, \epsilon_{2 i}=\epsilon_{1 i}, 0<\epsilon_{3 i}<\pi / 2,
\end{aligned}
$$

where $\lambda_{0 i}$ is a positive constant, $\boldsymbol{A}_{i}$ is defined in (16), and

$$
\begin{aligned}
& \lambda_{1 i}=\left(2\left|\varepsilon_{i} Y_{|v| v i}\right|+\left|Y_{|r| v i}\right|+\left|Y_{|v| r i}\right|\right) / m_{22 i}, \\
& \lambda_{2 i}=\left(\varepsilon_{i}^{2}\left|Y_{|v| v i \mid}\right|+\varepsilon_{i}\left(\left|Y_{|r| v i \mid}\right|+\left|Y_{|v| r i}\right|\right)+\left|Y_{|r| r i \mid}\right|\right) / m_{22 i},
\end{aligned}
$$

with $\varepsilon_{i}$ given just below (14). In particular, there are no collisions between any ships for all $t \geq t_{0} \geq 0$, the closed loop system (45) is forward complete, and the ships' trajectories track their reference trajectories in the sense of (23).

Proof. See Appendix A.

\section{Simulation results}

We simulate formation tracking control of a group of $N=$ 7 identical underactuated container ships, with a length of $230.66 \mathrm{~m}$ and a beam of $32 \mathrm{~m}$. The non-dimensional parameters of the ship taken from Perez and Blanke (2002) are (multiplied by $10^{-5}$ ): $m_{i}=750.81, x_{g i}=-200, I_{z i}=43.25$, $X_{u i}=-124.4, Y_{\dot{v} i}=-878, Y_{\dot{r} i}=-48.1, N_{\dot{ }}=-30, X_{u i}=-226.5$, $X_{|u| u i}=-64.4, Y_{v i}=-725, Y_{|v| v i}=-5801.5, Y_{|r| v i}=-1192.7$, $Y_{r i}=118.2, Y_{|v| r i}=-409.4, Y_{|r| r i}=0, N_{v i}=-300, N_{|v| v i}=-712.9$, $N_{|r| v i}=-174.7, N_{r i}=0, N_{|| \mid r i}=-778.8, N_{|r| r i}=0$.

The ships are initially positioned uniformly on a circle with a radius of $R_{o}=6$, the same heading of 1.8 , and zero velocities. We choose $\boldsymbol{d}_{i}=\left[\begin{array}{lll}m_{11 i} & m_{22 i} & m_{33 i}\end{array}\right]^{T}$ for all $i \in \mathbb{N}$, and $\boldsymbol{p}_{i d}=\boldsymbol{p}_{o d}\left(s_{i d}\right)+\boldsymbol{l}_{i}, \psi_{i d}=\arctan \left(y_{o d}^{\prime} / x_{o d}^{\prime}\right)$, where $\boldsymbol{p}_{o d}\left(s_{i d}\right)=$ $\left[x_{o d}\left(s_{i d}\right) y_{o d}\left(s_{i d}\right)\right]^{T}, x_{o d}^{\prime}=\frac{\partial x_{o d}}{\partial s_{i d}}, y_{o d}^{\prime}=\frac{\partial y_{o d}}{\partial s_{i d}}$, and $s_{i d}\left(t_{0}\right)=0$. The reference vectors $\boldsymbol{l}_{i}$ and $\boldsymbol{p}_{o d}\left(s_{i d}\right)$ are chosen such that we perform both linear and circular formations. In particular, for the non-dimensional time $t \leq 5$ we choose $\boldsymbol{l}_{i}=[0-1.25(i-1)]^{T}$ and $\boldsymbol{p}_{o d}=\left[s_{i d} 0\right]^{T}$ and $\dot{s}_{o d}=5$, i.e., the reference trajectory $\boldsymbol{p}_{o d}$ is a straight-line. For the non-dimensional time $t>5$, we take $\boldsymbol{l}_{i}=-1.25(i-1)\left[-\cos \left(s_{i d}\right) \cos \left(s_{i d}+\pi / 2\right)\right]^{T}$ and $\boldsymbol{p}_{o d}=10\left[\sin \left(s_{i d}\right) \cos \left(s_{i d}\right)\right]^{T}$ and $\dot{s}_{o d}=5$, i.e., the reference trajectory $\boldsymbol{p}_{\text {od }}$ is a circle with a radius of 10 .

To satisfy the conditions (19), (22), (31), (38), and (46), the control design constants are chosen as $\epsilon_{1 i}=\epsilon_{2 i}=0.2, \epsilon_{3 i}=$ $0.1, R_{i}=3, a_{i}=1, b_{i}=0.3, \boldsymbol{K}_{1}=\operatorname{diag}(0.2,0.2), k_{2}=0.2$, $c_{1}=10, c_{2}=5, k_{i d}=2, k_{i j}=5, a_{i j}=0, b_{i j}=0.25, a_{i j d}=0.3$, $b_{i j d}=0.35$, and $\boldsymbol{K}_{0 i}=\operatorname{diag}(3,3,3)$.

Several snapshots of the ships and their trajectories in $x y$ plane are plotted in Figure 5. The little dark color circular disk indicates the head of the ship. The representative distance $\chi_{i j}^{*}=\left(\prod_{j \in \mathbb{N}, j \neq i} \chi_{i j}\right)^{1 / 26}$ is plotted in the $1^{\text {st }}$ sub-figure of Figure 6. It is seen that $\chi_{i j}^{*}>0$, i.e., $\chi_{i j}>0$, hence $\Delta_{i j}>1$ for all $(i, j) \in \mathbb{N}, i \neq j$ implying no collision between any ships. The tracking errors $\left[x_{e} y_{e} \psi_{e}\right]^{T}=\boldsymbol{\eta}_{i}^{*}-\boldsymbol{\eta}_{i d}$ are plotted in the $2^{\text {nd }}, 3^{\text {rd }}, 4^{\text {th }}$ sub-figure of Figure 6 . The control inputs $\tau_{u}=\left[\tau_{u 1}, \ldots, \tau_{u N}\right]^{T}, \tau_{r}=\left[\tau_{r 1}, \ldots, \tau_{r N}\right]^{T}$, and $\dot{\alpha}=\left[\dot{\alpha}_{1}, \ldots, \dot{\alpha}_{N}\right]^{T}$ are plotted in the $5^{\text {th }}, 6^{\text {th }}, 7^{\text {th }}$ sub-figure of Figure 6 . It is noted that the tracking errors converge to a ball centered at the origin instead of zero since our proposed formation controller solved the practical formation control problem, see the coordinate transformation (14). High frequency oscillations in the controls $\tau_{u i}, \tau_{r i}$, and the additional control $\dot{\alpha}_{i}$ during the transient (non-dimensional) time can be reduced by tuning the control gains $\boldsymbol{K}_{1}, k_{2}, c_{1}, c_{2}, \epsilon_{1 i}, \epsilon_{2 i}$, and $\epsilon_{3 i}$ with a tradeoff of larger tracking errors and longer transient response time. From Figure 5 , it can be seen that when the lateral distance between ships is small, the proposed formation controller forces the ships to turn their heading a (plus or minus) small angle and to move in the surge direction backward or forward at the same time, i.e., the ships move in a zigzag way, to prevent collision between them.

Finally, it should be stressed that if one uses a circular disk to bound the ship in this simulation, the radius of the bounding circle must not be less than $a_{i}=1$. Consequently, no desired formation as the one in the above simulation can be achieved because $\left\|\boldsymbol{l}_{i}-\boldsymbol{l}_{i-1}\right\|=1.25$, which is smaller than $a_{i}+a_{i-1}=2$, for all $i=2, \ldots, N$.

\section{Conclusions}

The keys to success of the proposed formation control design included the separation condition for ellipses, the coordinate changes, and the potential functions. An extension of the 


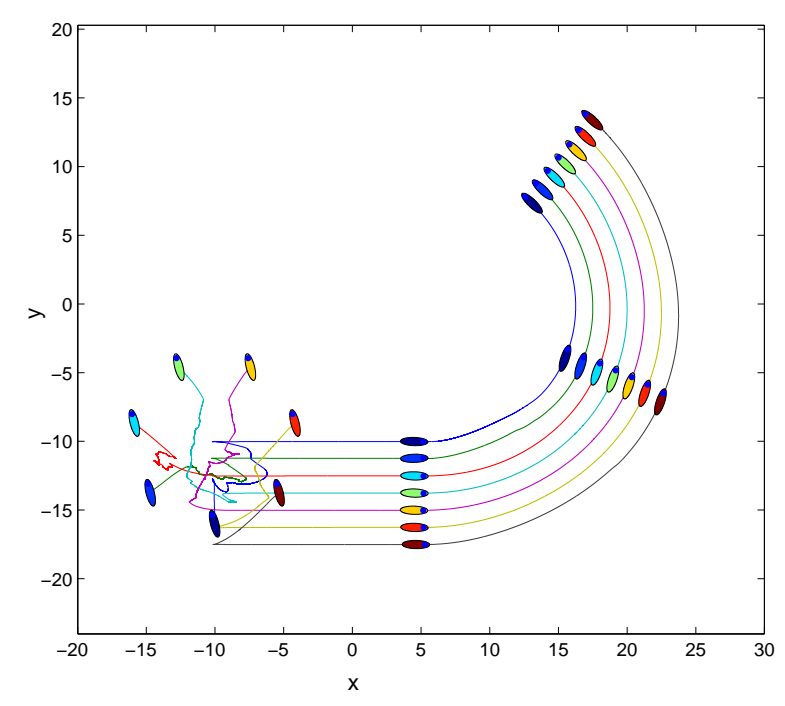

Figure 5: Snapshots of the ships and their trajectories in $X Y$ plane.
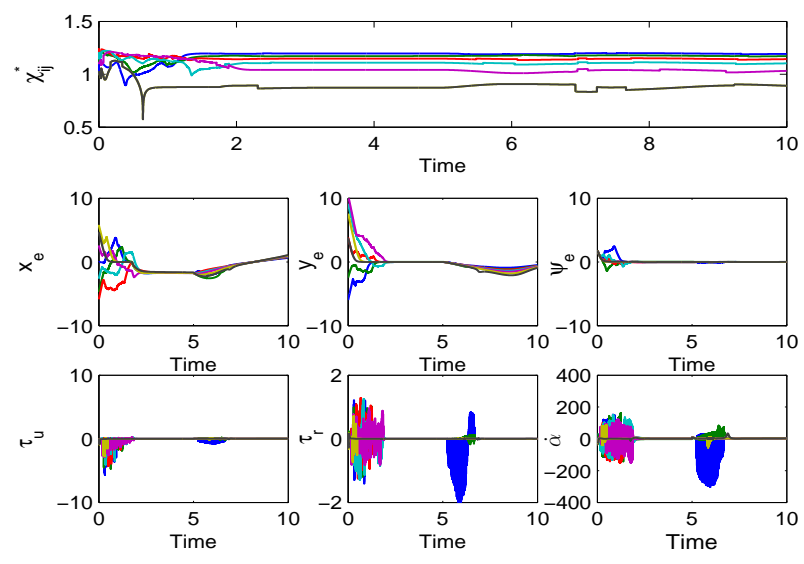

Figure 6: Representative $\chi_{i j}^{*}$, tracking errors, and control inputs.

proposed formation control design in this paper to provide a formation control system for a group of underactuated underwater vehicles is under consideration.

\section{Appendix A. Proof of Theorem 4.1}

1) Proof of complete forwardness of the closed loop system: Consider the Lyapunov function candidate

$$
W_{1}=\varphi_{I I}+\frac{\sigma_{1}}{2} \sum_{i=1}^{N} \boldsymbol{d}_{i e}^{T} \boldsymbol{d}_{i e}+\sigma_{2} \sum_{i=1}^{N}\left(\sqrt{1+\bar{v}_{i}^{2}}-1\right)
$$

where $\sigma_{1}$ and $\sigma_{2}$ are positive constants to be chosen later. Differentiating (A.1) using (44) and (45) results in

$$
\begin{gathered}
\dot{W}_{1}=\sum_{i=1}^{N}\left[-c_{1} \boldsymbol{\Omega}_{p i}^{T} \boldsymbol{\Phi}\left(\boldsymbol{\Omega}_{p i}\right)-c_{1} \Omega_{\psi_{i}} \Phi\left(\Omega_{\psi_{i}}\right)-c_{2} u_{i e}^{2}-c_{2} r_{i e}^{2}+\right. \\
\left.u_{i e}\left(\frac{\vartheta_{1 i e}}{m_{11 i}}-\frac{\partial \varpi_{u i}}{\partial \bar{v}_{i}} \frac{\vartheta_{2 i e}}{m_{22 i}}\right)+r_{i e}\left(\frac{m_{23 i}}{\Delta_{i}} \vartheta_{3 i e}-\frac{\partial \varpi_{r i}}{\partial \bar{v}_{i}} \frac{\vartheta_{2 i e}}{m_{22 i}}\right)\right]- \\
\sigma_{1} \sum_{i=1}^{N} \boldsymbol{d}_{i e}^{T} \boldsymbol{K}_{0 i} \boldsymbol{d}_{i e}+\sigma_{2} \sum_{i=1}^{N} \frac{1}{\sqrt{\bar{v}_{i}^{2}+1}}\left[\left(\frac{Y_{|v| v i}}{m_{22 i}}+\frac{m_{11 i}}{m_{22 i}} \times\right.\right. \\
\left.\frac{\left|f_{1 i}^{\prime}\right|+\left|f_{2 i}^{\prime}\right|}{\operatorname{det}\left(\boldsymbol{A}_{i}\right)}+\frac{\left|f_{3 i}^{\prime}\right|\left(\lambda_{1 i}+4 \lambda_{2 i}\right)}{\operatorname{det}\left(\boldsymbol{A}_{i}\right)}\right)\left|\bar{v}_{i}\right| \bar{v}_{i}^{2}+Y_{v i} m_{22 i}^{-1} \bar{v}_{i}^{2}+A_{1 i} \bar{v}_{i}^{2}+ \\
\left.A_{0 i}\right]+A_{2 i}\left|\Phi\left(\Omega_{\psi i}\right)\right|^{2}+A_{3 i}|| \boldsymbol{\Phi}\left(\boldsymbol{\Omega}_{p i}\right) \|^{2}+A_{4 i} u_{i e}^{2}+A_{5 i} r_{i e}^{2},
\end{gathered}
$$

where $\lambda_{1 i}$ and $\lambda_{2 i}$ are defined in (47), and $A_{l i}, l=0, \cdots, 5$ are nonnegative constants. With $\epsilon_{i}, i=1-3$ in (46), a calculation shows from (A.2) that $\dot{W}_{1} \leq C_{0}$ with $C_{0}$ a nonnegative bounded constant, i.e., (45) is forward complete.

2) Proof of no collisions: We consider the function $W_{2}=$ $\varphi_{I I}+\frac{\sigma_{1}}{2} \sum_{i=1}^{N} \boldsymbol{d}_{i e}^{T} \boldsymbol{d}_{i e}$ whose derivative along the solutions of (45) satisfies $\dot{W}_{2} \leq-\omega$ with $\omega:=\sum_{i=1}^{N}\left[c_{1} \boldsymbol{\Omega}_{p i}^{T} \boldsymbol{\Phi}\left(\boldsymbol{\Omega}_{p i}\right)+\right.$ $\left.c_{1} \Omega_{\psi_{i}} \Phi\left(\Omega_{\psi_{i}}\right)+c_{2}^{*} u_{i e}^{2}+c_{2}^{*} r_{i e}^{2}+c_{3}^{*}\left\|\boldsymbol{d}_{i e}\right\|^{2}\right]$, where $c_{2}^{*}=c_{2}-\epsilon$, $c_{3}^{*}=\sigma_{1} \lambda_{\min }\left(\boldsymbol{K}_{0 i}\right)-1 / \epsilon$ with $\lambda_{\min }\left(\boldsymbol{K}_{0 i}\right)$ the minimum eigenvalue of $\boldsymbol{K}_{0 i}$, and $\epsilon$ a positive constant. Picking $\epsilon$ and $\sigma_{1}$ such that the constants $c_{2}^{*}$ and $c_{3}^{*}$ are strictly positive, we have $\dot{W}_{2} \leq 0$. Hence $W_{2}(t) \leq W_{2}\left(t_{0}\right)$ for all $t \geq t_{0} \geq 0$. From the condition (22) and Property 3) of $\beta_{i j}$, we have $W_{2}\left(t_{0}\right)$ is bounded by a positive constant. Boundedness of $W_{2}\left(t_{0}\right)$ implies that of $W_{2}(t)$. As a result, $\beta_{i j}\left(\chi_{i j}(t)\right)$ must be smaller than some positive constant for all $t \geq t_{0} \geq 0$. From properties of $\beta_{i j}, \chi_{i j}(t)>0$ or $\Delta_{i j}(t)>1$, i.e., there are no collisions between any ships for all $t \geq t_{0} \geq 0$. Moreover, $W_{2}(t) \leq W_{2}\left(t_{0}\right)$ also implies that $\left\|\boldsymbol{\eta}_{i}-\boldsymbol{\eta}_{i d}\right\|$ is bounded.

3) Equilibrium set: Integrating both sides of $\dot{W}_{2} \leq-\omega$ yields $\int_{0}^{\infty} \omega(t) d t=W_{2}\left(t_{0}\right)-W_{2}(\infty) \leq W_{2}\left(t_{0}\right)$. Indeed, the function $\omega(t)$ is scalar, nonnegative and differentiable. Now differentiating $\omega(t)$ along the solutions of (45) and using Properties 2) and 5) of $\beta_{i j}$ given in (29) readily show that $\left|\frac{\mathrm{d} \omega(t)}{\mathrm{d} t}\right| \leq M \omega(t)$ with $M$ being a positive constant. Therefore Lemma 2 in Do (2007) results in $\lim _{t \rightarrow \infty} \omega(t)=0$, which implies from expressions of $\omega(t), \boldsymbol{\Omega}_{p i}$ and $\Omega_{\psi_{i}}$ in (35), and boundedness of $\left\|\boldsymbol{\eta}_{i}-\boldsymbol{\eta}_{i d}\right\|$ that $\lim _{t \rightarrow \infty}\left(\boldsymbol{K}_{1}\left(\boldsymbol{p}_{i}(t)-\boldsymbol{p}_{i d}(t)\right)+\sum_{j \neq i} \beta_{j i}^{\prime}(t) \boldsymbol{\Gamma}_{i j}(t)\right)=0$ and $\lim _{t \rightarrow \infty}\left(k_{2}\left(\psi_{i}(t)-\psi_{i d}(t)\right)+\sum_{j \neq i} \beta_{j i}^{\prime}(t) \Xi_{i j}(t)\right)=0$. These limits imply that $\boldsymbol{\eta}(t)=\left[\boldsymbol{\eta}_{1}^{T}(t), \ldots, \boldsymbol{\eta}_{N}^{T}(t)\right]^{T}$ can tend to $\boldsymbol{\eta}_{d}=$ $\left[\boldsymbol{\eta}_{1 d}^{T}, \ldots, \boldsymbol{\eta}_{N d}^{T}\right]^{T}$, since $\beta_{i j}^{\prime}(t)=0$ at $\boldsymbol{\eta}_{i}=\boldsymbol{\eta}_{i d}$ and $\boldsymbol{\eta}_{j}=\boldsymbol{\eta}_{j d}$ (see, Property 1) of $\beta_{i j}$ ), or tend to a vector denoted by $\boldsymbol{\eta}_{c}=$ $\left[\boldsymbol{\eta}_{1 c}^{T}, \ldots, \boldsymbol{\eta}_{N c}^{T}\right]^{T}$ with $\boldsymbol{\eta}_{i c}=\left[x_{i c} y_{i c} \psi_{i c}\right]^{T}$ as the time goes to infinity, i.e., the equilibrium sets can be $\Xi_{d}$ containing $\boldsymbol{\eta}_{d}$ or $\Xi_{c}$ containing $\boldsymbol{\eta}_{c}$. The vector $\boldsymbol{\eta}_{c}$ is such that

$$
\begin{aligned}
& \boldsymbol{\Omega}_{p i c}=\left.\left(\boldsymbol{K}_{1}\left(\boldsymbol{p}_{i}-\boldsymbol{p}_{i d}\right)+\sum_{j \neq i} \beta_{j i}^{\prime} \boldsymbol{\Gamma}_{i j}\right)\right|_{\eta=\boldsymbol{\eta}_{c}}=0, \\
& \Omega_{\psi_{i c}}=\left.\left(k_{2}\left(\psi_{i}-\psi_{i d}\right)+\sum_{j \neq i} \beta_{j i}^{\prime} \Xi_{i j}\right)\right|_{\eta=\boldsymbol{\eta}_{c}}=0,
\end{aligned}
$$

for all $i \in \mathbb{N}$. To investigate stability properties of $\Xi_{d}$ and $\Xi_{c}$, we consider the first equation of (45), i.e.,

$$
\begin{aligned}
& \dot{\boldsymbol{p}}_{i}=-c_{1} \boldsymbol{\Phi}\left(\boldsymbol{\Omega}_{p i}\right)+\dot{\boldsymbol{p}}_{i d}+\boldsymbol{\Psi}_{p i}, \\
& \dot{\psi}_{i}=-c_{1} \Phi\left(\Omega_{\psi_{i}}\right)+\dot{\psi}_{i d}+\Psi_{\psi i},
\end{aligned}
$$

where $\boldsymbol{\Psi}_{p i}=\boldsymbol{A}_{i}\left[u_{i e} 0\right]^{T}+\boldsymbol{R}_{i}^{\prime}\left[f_{1 i} f_{2 i}\right]^{T} r_{i e}$ and $\Psi_{\psi i}=r_{i e}$. We treat $\boldsymbol{\Psi}_{p i}$ and $\Psi_{\psi i}$ as inputs to (A.4) instead of states. We now need to prove that $\Xi_{d}$ is locally asymptotically stable and that $\Xi_{c}$ is locally unstable.

4) Proof of $\Xi_{d}$ being asymptotically stable: Since $\left.\beta_{i j}^{\prime}\right|_{\eta=\eta_{d}}=0$ and $\left.\beta_{i j}^{\prime \prime}\right|_{\boldsymbol{\eta}=\boldsymbol{\eta}_{d}}=0$, see Property 1) of the function $\beta_{i j}$ in (29), local asymptotic stability of the equilibrium set $\Xi_{d}$ follows from (A.4) by using the function $V_{d}=\frac{1}{2} \sum_{i=1}^{N}\left(\sqrt{\left\|\boldsymbol{p}_{i}-\boldsymbol{p}_{i d}\right\|^{2}+\left(\psi_{i}-\psi_{i d}\right)^{2}+1}-1\right)$ and $\lim _{t \rightarrow \infty}\left(\Psi_{p i}(t), \Psi_{\psi i}(t)\right)=0$.

5) Proof of $\Xi_{c}$ being unstable: Let $\mathbb{N}^{*}$ be the set of the ships such that if the ships $i$ and $j$ belong to the set $\mathbb{N}^{*}$ then $\chi_{i j}<b_{i j d}$, and $N^{*}$ be the size of $\mathbb{N}^{*}$. In $\mathbb{N}^{*}$, we have $\dot{\boldsymbol{\eta}}_{i d}=0$. From (A.3) we have $\sum_{(i, j) \in \mathbb{N}^{*}} \boldsymbol{p}_{i j c}^{T}\left(\boldsymbol{\Omega}_{p i c}-\boldsymbol{\Omega}_{p i c}\right)$, which is expanded as:

$$
\sum_{(i, j) \in \mathbb{N}^{*}} \boldsymbol{p}_{i j c}^{T} \boldsymbol{L}_{i j} \boldsymbol{p}_{i j c}=\sum_{(i, j) \in \mathbb{N}^{*}} \boldsymbol{p}_{i j c}^{T} \boldsymbol{K}_{1} \boldsymbol{p}_{i j d}
$$


where $\boldsymbol{L}_{i j}=\boldsymbol{K}_{1}+N^{*}\left(\beta_{j i c}^{\prime} \boldsymbol{\Gamma}_{i j c}^{*}+\beta_{i j c}^{\prime} \boldsymbol{\Gamma}_{j i c}^{*}\right)$. Since $\left\|\boldsymbol{p}_{i j d}\right\|$ and $\left\|\boldsymbol{p}_{i j c}\right\|$ are bounded, (A.5) indicates that $\lim _{\lambda_{\max }\left(\boldsymbol{K}_{1}\right) \rightarrow 0} \beta_{j i c}^{\prime}=-\infty$. Hence there exists $\boldsymbol{K}_{1}$ with a sufficiently small $\lambda_{\max }\left(\boldsymbol{K}_{1}\right)$ such that $\boldsymbol{L}_{i j}$ is negative definite for some $(i, j)$ with $i \neq j$. Let $\mathbb{N}^{* *} \subset \mathbb{N}^{*}$ be a nonempty set such that for all $(i, j) \in \mathbb{N}^{* *}, i \neq j$, $\boldsymbol{L}_{i j}$ is negative definite. Moreover, we define a set $U$ such that

$$
U=\left\{\left(\boldsymbol{p}_{i j}, \psi_{i}, \psi_{j}\right) \in B_{r} \mid U_{1} \leq 0, U_{2} \leq 0, U_{3} \leq 0,\right.
$$

for all $(i, j) \in \mathbb{N}^{* *}, i \neq j$, where $U_{1}=\cup_{i=1}^{N^{* *}}\left(-\left(\psi_{i}-\psi_{i c}\right) \Phi\left(\Omega_{\psi_{i}}\right)\right)$, $U_{2}=\cup_{(i, j) \in \mathbb{N}^{* *}}\left(\left(\overline{\boldsymbol{Q}}_{i j}\left(\boldsymbol{p}_{i j}-\boldsymbol{p}_{i j c}\right)\right)^{T}\left(\overline{\boldsymbol{Q}}_{i j}^{-T}\left(\beta_{j i}^{\prime} \boldsymbol{\Gamma}_{i j}^{*}-\beta_{j i c}^{\prime} \boldsymbol{\Gamma}_{i j c}^{*}\right) \overline{\boldsymbol{Q}}_{i j}^{-1}\right) \overline{\boldsymbol{Q}}_{i j} \boldsymbol{p}_{i j}\right)$, and $U_{3}=\cup_{(i, j) \in \mathbb{N}^{* *}}\left(\left(\overline{\boldsymbol{Q}}_{j i}\left(\boldsymbol{p}_{j i}-\boldsymbol{p}_{j i c}\right)\right)^{T}\left(\overline{\boldsymbol{Q}}_{j i}^{-T}\left(\beta_{i j}^{\prime} \boldsymbol{\Gamma}_{j i}^{*}-\beta_{i j c}^{\prime} \boldsymbol{\Gamma}_{j i c}^{*}\right) \overline{\boldsymbol{Q}}_{j i}^{-1}\right) \overline{\boldsymbol{Q}}_{j i} \boldsymbol{p}_{j i}\right)$, where $i \neq j$, and $\overline{\boldsymbol{Q}}_{i j}=\boldsymbol{Q}_{i j} \boldsymbol{R}_{i}$. It can be readily proved that the set $U$ is non-empty. In $U$, we consider the function

$$
\bar{V}_{c}^{* *}=\frac{1}{2} \sum_{(i, j) \in \mathbb{N}^{* *}} \sqrt{\left\|\boldsymbol{p}_{i j}-\boldsymbol{p}_{i j c}\right\|^{2}+1}+\frac{1}{2} \sum_{i=1}^{N^{* *}} \sqrt{\left(\psi_{i}-\psi_{i c}\right)^{2}+1},
$$

whose derivative along the solutions of (A.4) satisfies

$$
\dot{\bar{V}}_{c}^{* *} \geq-c_{1} \sum_{(i, j) \in \mathbb{N}^{* *}} \frac{\left(\boldsymbol{p}_{i j}-\boldsymbol{p}_{i j c}\right)^{T} \boldsymbol{H}_{p i j} \boldsymbol{T}_{i j}\left(\boldsymbol{p}_{i j}-\boldsymbol{p}_{i j c}\right)}{\sqrt{\left\|\boldsymbol{p}_{i j}-\boldsymbol{p}_{i j c}\right\|^{2}+1}}+\Theta_{c},
$$

where $\boldsymbol{H}_{p i j}=\operatorname{diag}\left(\frac{\Phi\left(\Omega_{x i}\right)-\Phi\left(\Omega_{x j}\right)}{\Omega_{x i}-\Omega_{x j}}, \frac{\Phi\left(\Omega_{y i}\right)-\Phi\left(\Omega_{y j}\right)}{\Omega_{y i}-\Omega_{y j}}\right), \boldsymbol{T}_{i j}=\boldsymbol{K}_{1}+$ $N^{* *}\left(\beta_{j i c}^{\prime} \boldsymbol{\Gamma}_{i j c}^{*}+\beta_{i j c}^{\prime} \boldsymbol{\Gamma}_{j i c}^{*}\right)$ with $\beta_{i j c}^{\prime}=\left.\beta_{i j}^{\prime}\right|_{\eta=\eta_{c}}, \boldsymbol{\Gamma}_{i j c}^{*}=\left.\boldsymbol{\Gamma}_{i j}^{*}\right|_{\boldsymbol{\eta}=\boldsymbol{\eta}_{c}}, \boldsymbol{p}_{i j c}=$ $\left.\boldsymbol{p}_{i j}\right|_{\boldsymbol{\eta}=\boldsymbol{\eta}_{c}}$, and $\Theta_{c}=\sum_{(i, j) \in \mathbb{N}^{* *}} \frac{\left(\boldsymbol{p}_{i j}-\boldsymbol{p}_{i j c}\right)^{T} \boldsymbol{\Psi}_{p i j}}{\sqrt{\left\|\boldsymbol{p}_{i j}-\boldsymbol{p}_{i j}\right\|^{2}+1}}+\sum_{i=1}^{N^{* *}} \frac{\left(\psi_{i}-\psi_{i c}\right) \Psi_{\psi i}}{\sqrt{\left(\psi_{i}-\psi_{i c}\right)^{2}+1}}$. Since $\boldsymbol{H}_{p i j}$ is positive definite, $\boldsymbol{T}_{i j}$ is negative definite, and $\lim _{t \rightarrow \infty} \Theta_{c}(t)=0$, Chetaev's Theorem (Theorem 4.3 in Khalil (2002)) implies that $\Xi_{c}$ is unstable. Since we have already proved that the errors $\left(\boldsymbol{\eta}_{i}(t)-\boldsymbol{\eta}_{i d}(t)\right),\left(\psi_{i}(t)-\psi_{i d}(t)\right), u_{i e}(t), r_{i e}(t)$ and $\boldsymbol{d}_{i e}(t)$ asymptotically converge to zero, boundedness of $\bar{v}_{i}$ directly follows from (A.2).

\section{References}

Borhaug, E., Pavlov, A., Panteley, E., Pettersen, K.Y., 2010. Straight line path following for formations of underactuated marine surface vessels. IEEE Transactions on Control Systems Technology, in press .

Borhaug, E., Pavlov, A., Pettersen, K.Y., 2006. Cross-track formation control of underactuated surface vessels. Proceedings of the 45th IEEE Conference on Decision and Control , 5955-5961.

Cui, R., Ge, S.S., Ho, B.V., Choo, Y., 2010. Leaderfollowerformationcontrolofunderactuatedautonomous underwater vehicles. Ocean Engineering, In press .

Do, K.D., 2007. Bounded controllers for formation stabilization of mobile agents with limited sensing ranges. IEEE Transactions on Automatic Control 52, 569-576.

Do, K.D., 2008. Formation tracking control of unicycle-type mobile robots with limited sensing ranges. IEEE Transactions on Control Systems Technology 16, 527-538.

Do, K.D., 2010. Practical control of underactuated ships. Ocean Engineering 37, 1111-1119.

Do, K.D., 2011. Formation control of multiple elliptical agents with limited sensing ranges. Automatica, Submitted .

Do, K.D., Pan, J., 2009. Control of Ships and Underwater Vehicles: Design for Underactuated and Nonlinear Marine Systems. Springer.

Dong, W., Farrell, J.A., 2008. Formation control of multiple underactuated surface vessels. IET Control Theory and Applications 2, 1077-1085.

Dong, W., Farrell, J.A., 2009. Decentralized cooperative control of multiple nonholonomic dynamic systems with uncertainty. Automatica 45, 706710.

Fahimi, F., 2007. Sliding-mode formation control for underactuated surface vessels. IEEE Transactions on Robotics 23, 617-622.

Fossen, T.I., 2002. Marine Control Systems. Marine Cybernetics, Trondheim, Norway.

Khalil, H., 2002. Nonlinear Systems. Prentice Hall.

Krstic, M., Kanellakopoulos, I., Kokotovic, P., 1995. Nonlinear and Adaptive Control Design. Wiley, New York.
Lapierre, L., Soetanto, D., Pascoal, A., 2003. Coordinated motion control of marine robots. The 6th IFAC Conf. Manoevering and Control of Marine Craft .

Morin, P., Samson, C., 2003. Practical stabilization of driftless systems on lie groups: the transverse function approach. IEEE Transactions on Automatic Control 48, 1496-1508.

Perez, T., Blanke, M., 2002. Mathematical ship modelling for marine applications. Technical report, Technical University of Denmark

Schoerling, D., Kleeck, C.V., Fahimi, F., Koch, C.R., Ams, A., Lober, P., 2010. Experimental test of a robust formation controller for marine unmanned surface vessels. Autonomous Robots 28, 213-230.

SNAME, 1950. The Society of Naval Architects and Marine Engineers. Nomenclature for Treating the Motion of a Submerged Body Through a Fluid. In: Technical and Research Bulletin No. 1-5. 